\title{
Le parcours de l'apprenti chercheur, entre solitude et collectif de recherche
}

Caroline Lejeune

\section{OpenEdition}

Journals

Édition électronique

URL : http://journals.openedition.org/developpementdurable/11318

DOI : 10.4000/developpementdurable.11318

ISSN : 1772-9971

Éditeur

Association DD\&T

Référence électronique

"Le parcours de l'apprenti chercheur, entre solitude et collectif de recherche », Développement durable et territoires [En ligne], Vol. 7, n² | Juillet 2016, mis en ligne le 28 juillet 2016, consulté le 24 septembre 2020. URL : http://journals.openedition.org/developpementdurable/11318; DOI : https://doi.org/ 10.4000/developpementdurable.11318

Ce document a été généré automatiquement le 24 septembre 2020.

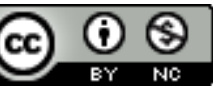

Développement Durable et Territoires est mis à disposition selon les termes de la licence Creative Commons Attribution - Pas d'Utilisation Commerciale 4.0 International. 


\title{
Le parcours de l'apprenti chercheur, entre solitude et collectif de recherche
}

\author{
Caroline Lejeune
}

1 Sur la ligne de départ, nous prenons le temps d'écouter les conseils des anciens et des jeunes chercheurs. Leurs anecdotes nous guident dans ce tout petit monde (Lodge, 1992) qu'est la recherche et on les retrouve au fil du temps dans les prises de notes de nos carnets de thèse (Rivière, 2015). Le doctorat est une étape unique dans la vie d'un chercheur et peut-être la plus importante. C'est un moment pendant lequel il peut vraiment prendre le temps de lire et de mener une quête scientifique sur son objet d'étude favori.

2 L'expérience doctorale est un rite académique et une phase d'adoubement par la communauté scientifique (Serre, 2015). Elle est souvent décrite comme une trajectoire individuelle et un processus de socialisation au monde de la recherche. Elle est d'abord connue pour être une épreuve où les doutes et la solitude animent ou perturbent notre quête (Chao et al., 2015). Combien d'entres nous se sont demandés s'ils allaient parvenir au bout de l'aventure?

3 Le doctorant est à la fois un étudiant en formation, un apprenti chercheur et un futur collègue. Il doit donc rapidement apprendre à porter ces différentes casquettes (Collectif TMC, 2015) : il mène sa recherche avec engagement, dans l'incertitude de son insertion professionnelle et doit anticiper un rôle futur, celui du métier d'enseignantchercheur. Il doit donc être sympathique, s'engager et s'investir dans son laboratoire ainsi que participer à la vie de l'université et de la recherche. Pourtant le fonctionnement vertical du milieu universitaire n'hésitera pas à lui rappeler qu'il n'est, encore, qu'un " étudiant », avec toute la violence symbolique et sociale que ce mépris comporte.

4 Le doctorat n'est pas qu'une quête individuelle. Le jeune chercheur peut aussi s'épanouir dans des collectifs de recherche. Mais entendons-nous. Il ne faut pas y voir une «logique de réseau» qui sert à promouvoir le seul intérêt individuel et à développer une stratégie personnelle. Même s'il n'est pas rare que le collectif soit une 
voie vers la promotion des carrières et des intérêts privés, cette «trajectoire collective " est aussi l'occasion pour un jeune chercheur de s'inscrire dans un projet scientifique collectif et partagé. Ce projet, parce qu'il rassemble, crée un environnement favorable à l'évolution des recherches sur un objet scientifique particulier. Il permet aussi de tisser des relations de confiance, d'affinités scientifiques et mêmes amicales.

5 La revue Développement Durable et Territoires fait partie de ces projets collectifs. Et dans ce tout petit monde de la recherche, elle crée de nouvelles formes de collaboration entre les chercheurs et les doctorants autour d'un objet scientifique interdisciplinaire en sciences humaines et sociales. À mes yeux, ces collaborations sont primordiales dans l'animation de la recherche. Pourtant elles peinent encore à être reconnues dans le milieu universitaire.

6 La revue Développement Durable et Territoires présente comme spécificité de toujours interroger le sens de son projet (Villalba, Petit, 2015), le cadre de ses pratiques éditoriales et porte une réflexion sur la place des doctorants dans la revue. Elle instaure des relations horizontales entre les doctorants et les chercheurs; elle accorde sa confiance aux doctorants invités à participer aux activités de la revue et leur confie des responsabilités; elle leurs offre aussi une expérience dans le milieu de la publication scientifique. Ces derniers peuvent alors proposer des sujets scientifiques émergents et finalement contribuer simplement et dans la convivialité au projet de publication. Ils bénéficient ainsi de conseils, de soutiens bienveillants. La revue instaure finalement des collaborations scientifiques apaisées qui permettent à chacun de progresser. Alors, oui le doctorat peut-être vécu comme un parcours personnel mais la recherche prend son sens quand elle s'insère dans un projet collectif.

7 Ma thèse est aujourd'hui terminée (enfin !) et je clôture cette étape avec cet éditorial estival. Je profite de l'occasion pour remercier les fondateurs de la revue, le directeur de la publication, les responsables de rubriques, les membres des trois comités, les coordinateurs de dossiers, de m'avoir initiée au monde de la publication scientifique et à son exigence, de m'avoir invitée à partager le projet de «DDT » et de m'avoir permis de découvrir l'immense travail collectif pour sortir chaque nouveau numéro.

8 Je n'ai plus qu'à vous souhaiter de bonnes vacances avec beaucoup de sobriété (... énergétique bien-sûr). Mais avant cela, venez jeter un coup d'œil à notre nouveau numéro. Comme promis, il est dédié à la seconde partie du dossier "adaptation en tension» coordonné par Christelle Audouit, ingénieure de recherche au laboratoire TVES de l'Université de Lille, membre du comité de rédaction de la revue et à Julien Rebotier, chargé de recherches au CNRS et membre du laboratoire LISST de l'Université de Toulouse. La rubrique "Varia " nous propose encore de nombreux articles. Enfin quelques belles recensions d'ouvrages pourront peut-être vous accompagner durant vos vacances.

Bel été ! 


\section{BIBLIOGRAPHIE}

Collectif TMTC, « L'horizon vertical de la recherche », 2015, Socio-logos [En ligne], $\mathrm{n}^{\circ}$ 10, URL : http://socio-logos.revues.org/2975.

Chao M., Monini C., Munck S., Thomas S., Rochot J., Van de Velde C., 2015, « Les expériences de la solitude en doctorat. Fondements et inégalités ", Socio-logos [En ligne], n 10, URL : http://sociologos.revues.org/2975.

Serre S., 2015, « Être doctorant-e. Socialisations, contextes, trajectoires », Socio-logos [En ligne], $\mathrm{n}^{\circ}$ 10, URL : http://socio-logos.revues.org/2975.

Lodge D., 1992, Un tout petit monde, Paris, Payot \& Rivages.

Rivière T., 2015, Carnets de thèse, Paris, Le Seuil.

Villalba B. et Petit O., 2014, «Quinze ans de recherches sur l'interface entre développement durable et territoires. Un bilan réflexif ", Développement durable et territoires, vol. 5, $\mathrm{n}^{\circ}$ 3, http:// developpementdurable.revues.org/10487, DOI : 10.4000/developpementdurable.10487. 